\title{
Geopotential determination based on a direct clock comparison using two-way satellite time and frequency transfer
}

\author{
Wen-Bin Shen ${ }^{1,2, *}$, Xiao Sun ${ }^{1}$, Chenghui Cai ${ }^{1},{\text { Kuangchao } \mathrm{Wu}^{1} \text {, and Ziyu Shen }}^{1}$ \\ ${ }^{1}$ Department of Geophysics, School of Geodesy and Geomatics/Key Laboratory of Geospace Environment and Geodesy of \\ Ministry of Education, Wuhan University, Wuhan, China \\ ${ }^{2}$ State Key Laboratory of Information Engineering in Surveying, Mapping and Remote Sensing, Wuhan University, Wuhan, China
}

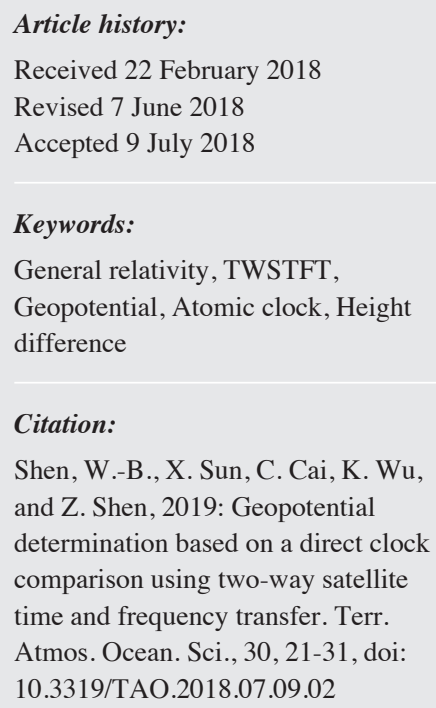

\begin{abstract}
According to Einstein's general relativity theory, a precise (atomic) clock runs faster at a position with higher gravitational potential than a clock at a position with lower potential. Here, we provide a direct clock comparison using a fixed hydrogen clock and a portable hydrogen clock for geopotential determination based on the two-way satellite time and frequency transfer (TWSTFT) technique. After synchronizing the two hydrogen clocks at positions at the same height, the clocks were compared for a height difference of $22 \mathrm{~m}$ over a period of 14 days using the TWSTFT technique. Interpretation of the observed time elapse implies a height difference of $175 \mathrm{~m}+/-59 \mathrm{~m}$, which matches the frequency stability of our instruments. This study and relevant experiments provide a way of determining the geopotential using ultrahigh precise clocks based on the TWSTFT technique in the near future.
\end{abstract}

\section{INTRODUCTION}

Gravity potential (geopotential) plays an important role in geodesy. Conventionally, we combine leveling and gravimetry to determine the geopotential (Hofmann-Wellenhof and Moritz 2006), which is referred to as the classic method. The classic method has at least two drawbacks: the error accumulates as the length of the measuring line increases, and it is difficult or impossible to transfer the orthometric height with high accuracy between two points located in mountainous areas or across the sea (Shen et al. 2016a, b).

To overcome the drawbacks of the classic method, Bjerhammar (1985) put forward an idea to determine the geopotential using a clock transportation approach (Shen et al. 2009) based on the general relativity theory (GRT) (Einstein 1915). According to GRT, in a gravitational field, a clock at a position with higher geopotential runs faster than one at a lower geopotential. That is accounted for gravitational redshift, which has been confirmed at different ac-

\footnotetext{
* Corresponding author

E-mail:wbshen@sgg.whu.edu.cn
}

curacy levels by various physicists (Pound and Rebka 1959, 1960a, b, 1965; Hafele and Keating 1972; Alley 1979; Vessot et al. 1980; Turneaure et al. 1983; Krisher el al. 1993). Later, Shen et al. (1993) suggested that the geopotential could be determined by the gravity frequency shift due to the gravitational redshift effect. In addition, Shen et al. $(1993,2011)$ put forward a new approach, gravity frequency shift approach, suggesting that Global Navigation Satellite System (GNSS) satellites could be used as inter-transmitters to transfer frequency signals, and in this way, the geopotential difference between two stations on the ground (even separated by the ocean) can be determined. This technique is also named chronometric leveling (Delva and Lodewyck 2013; Mai and Müller 2013). Chronometric leveling in clock networks will provide completely independent and possibly very precise observations of the geopotential differences in specific points (Flury 2016), which may help determine gravity field and establish world height system (Shen 2017; Müller et al. 2018; Shen et al. 2018a).

To test gravitational redshift effect, Chou et al. (2010) 
set two optical clocks in separated laboratories which were connected through a 75-m length of phase-stabilized optical fiber, and their experimental result of height difference $37 \pm 15 \mathrm{~cm}$ agrees well with the known value of $33 \mathrm{~cm}$. Shen and Peng (2012) and Shen (2013a, b) proposed optical frequency fiber transfer (OFFT) approach to determine the geopotential. First, two optical clocks should be synchronized at same site. Then they are transported to two different places. Just using the optical fiber frequency transfer comparison technique, one could determine the frequency shift $\Delta f$. In this way, we could determine the geopotential difference by gravity redshift equation. Later Lisdat et al. (2016) and Grotti et al. (2018) successively provided relevant experimental results which confirmed the OFFT approach (Shen and Peng 2012; Shen 2013a, b; Shen et al. 2018b).

However, the chronometric leveling is better expected for long-distance height differences (Flury 2016). Kopeikin et al. (2016) conducted an experiment using two hydrogen clocks in the Altai Mountains area. They compared the clock rate of two stations separated by a distance of about $35 \mathrm{~km}$ based on the time elapse between the two clocks. The comparison was carried out using pseudo-range phase measurements of GPS/GLONASS navigation satellites, and their result is $859 \mathrm{~m}$, with an uncertainty of $64 \mathrm{~m}$, comparing with the actual height difference of $725 \mathrm{~m}$.

In recent years, with quick development of high-precision clock manufacturing technology, the optical-atomic clocks (OACs) with relative instability around $10^{-18}$ in several hours and inaccuracy of $10^{-18}$ level have been generated in the laboratory (Hinkley et al. 2013; Bloom et al. 2014; Ushijima et al. 2015; Shen et al. 2017). Now an optical clock network is to be constructed, aims to compare and link the optical atomic clocks with the highest accuracy, and OACs with such precision level are promising to be installed on satellites in the near future (Riehle 2017). Theoretically, if clocks or oscillators with an inaccuracy of $1 \times 10^{-18}$ are available, we may determine the height difference with an uncertainty of $1 \mathrm{~cm}$ (Shen et al. 2016a, b, 2017,2018 b), providing a potential way to establish a world height system at $1 \mathrm{~cm}$ level.

Apart from GNSS satellites, geostationary satellites can also serve as a 'bridge'. The technique of two-way satellite time and frequency transfer (TWSTFT, in earlier documents, the term TWSTT) is a promising tool using geostationary satellites for accurate time and frequency transfer (Kirchner 1991; Jiang et al. 2013; Bauch 2015). Before GPS time transfer became popular in the 1980 s, researchers attempted to use geostationary telecommunication satellites to compare clocks, dating back to August 1960. In the earliest years, the two-way satellite time transfer uncertainty was approximately $10-100 \mathrm{~ns}$, but by the end of the 1970s, experiments proved that type A uncertainty [it was defined by JCGM 100 (2008) that this type can be evaluated by statistical methods] could be further improved to approximate- ly 0.2 ns. In 1989, the 11th CCDS (Consultative Committee for the Definition of the Second, now the Consultative Committee for Time and Frequency, CCTF) encouraged the use of two-way time transfer and suggested the creation of an ad-hoc working group on the TWSTFT at the BIPM (International Bureau of Weights and Measures). Later, ten laboratories actively intended to join the experimental plan. In August 1999, a TWSTFT link was introduced for clock comparison in UTC, and the time transfer uncertainty was greatly improved.

Experiments show that TWSTFT can compare time and frequency with high accuracy. In 2009, ten timing laboratories participated in an experiment to study the $1 \mathrm{MChip} / \mathrm{s}$ TWSTFT performance, and they obtained a short-term stability of $200-300 \mathrm{ps}$ at $1 \mathrm{~s}$ and $20-40 \mathrm{ps}$ when averaged over 128 s (Zhang and Parker 2009). In 2013, an experiment using carried-phase TWSTFT was performed between the two stations of NICT (National Institute of Information and Communications Technology) and PTB (PhysikalischTechnische Bundesanstalt), and a largely improved shortterm instability for a frequency transfer of $2 \times 10^{-13}$ at $1 \mathrm{~s}$ was achieved (Fujieda et al. 2014). During the same campaign, two ${ }^{87} \mathrm{Sr}$ lattice optical frequency standards operated respectively at NICT and PTB were compared. Based on a total measurement time of $83640 \mathrm{~s}$, relative frequency difference of $(0.8 \pm 1.6) \times 10^{-15}$ was obtained, where the statistical measurement uncertainty is the biggest contribution to the combined uncertainty (Bauch 2015). The current TWSTFT may theoretically attain a stability of $0.1 \mathrm{ps}$, with the prospect of reaching a long-term stability of $10^{-17}$ level (Jiang et al. 2013; Fujieda et al. 2016). And that is considered as a potential frequency transfer technique of optical clock networks (Riehle 2017). Kong et al. (2016) used TWSTFT observations released by BIPM to estimate the frequency difference of two stations, and obtained height differences with an accuracy of $10 \mathrm{~m}$. In our knowledge however, until now we did not find any literature that addressed the actual TWSTFT experiment for the chronometric leveling.

In this study, to further investigate the feasibility of clock comparison using TWSTFT, we conducted a clock comparison experiment at the Beijing Institute of Radio Metrology and Measurement (BIRMM), Beijing. In section 2, we demonstrate a solution for determining the geopotential via TWSTFT, and in section 3, we discuss error corrections. Section 4 describes our experiment, section 5 presents the data processing, and section 6 provides the results. Conclusions and relevant discussions are provided in section 7.

\section{METHODS}

\subsection{Geopotential Determination Based on Time Elapse Between Two Clocks}

According to GRT, in a gravity field, a clock at a position with a higher geopotential runs faster than one with 
a lower geopotential (Shen et al. 2009). Suppose there are two stations, A and B, with an orthometric height difference $\Delta H$. After a standard time $T$, accurate to $1 / c^{2}$, this time difference can be expressed as (Bjerhammar 1985; Shen et al. 1993; Pavlis and Weiss 2003; Dittus et al. 2007)

$\Delta t_{A B}=t_{B}-t_{A}=-\left(W_{B}-W_{A}\right) \frac{T}{c^{2}}$

where $W_{A}$ and $W_{B}$ denote the geopotentials at stations $\mathrm{A}$ and $\mathrm{B}$ respectively, and $c$ denotes the speed of light in vacuum. In the process of testing the Einstein equivalence principle, Eq. (1) has been confirmed to the $6.0 \times 10^{-5}$ level by a Gravity Probe-A (GPA) experiment using radio techniques (Vessot et al. 1980).

In plane areas, we have the following equation (Hofmann-Wellenhof and Moritz 2006)

$W_{B}-W_{A}=\Delta W_{A B} \approx-g \Delta H_{A B}=-g\left(H_{B}-H_{A}\right)$

where suppose $W_{A}$ is known, $g$ is a "mean value" of the Earth's gravity between $B$ and $B$ ' that is the intersection point of the plumb line of $B$ and the geopotential surface passing through $A$ (Shen et al. 2018b), referring to Fig. 1 . Thus, Eq. (1) can be written as:

$\Delta H_{A B}=\frac{\Delta t_{A B}}{T} \frac{c^{2}}{g}$

In this way, we can obtain the orthometric height difference
$\Delta H$ from the elapsed time difference $\Delta t$. As Eq. (3) shows, $\Delta H$ is proportional to $\Delta t / T$. We note that, for our experiment's purpose, the accuracy of Eq. (3) is sufficient. For a centimeter-level measurement, a more accurate formula is needed, as referred to Shen et al. (2017).

\subsection{Determination of Time Elapse of Two Clocks via TWSTFT}

The TWSTFT is based on the exchange of timing signals through geostationary telecommunication satellites, as schematically shown in Fig. 2, which illustrates various signal delays and elucidates how the time difference between the clocks at stations 1 and 2 can be determined.

For a standard TWSTFT system, the time-scale difference is given by the following two-way equation (ITU-R 2010)

$$
\begin{aligned}
& T S(1)-T S(2)= \\
& 0.5[T I(1)]-0.5[T I(2)]+0.5[S P T(1)-S P T(2)] \\
& -0.5[S C D(1)-S C U(1)]+0.5[S C D(2)-S C U(2)] \\
& +0.5[S P U(1)-S P D(1)]-0.5[S P U(2)-S P D(2)] \\
& +0.5[T X(1)-R X(1)]-0.5[T X(2)-R X(2)]
\end{aligned}
$$

where $T S(k)$ denotes the local time-scale, and $k$ stands for station $k(k=1,2)$; $\operatorname{TI}(k)$, the time interval reading, and the counter gate is opened by a 1PPS signal related to the local transmission signal and closed by a 1PPS signal related to the received signal, with short-form designation of 1PPSTX - 1PPSRX; TX $(k)$, the transmitter delay, including the modem delay; $R X(k)$, the receiver delay, including the modem

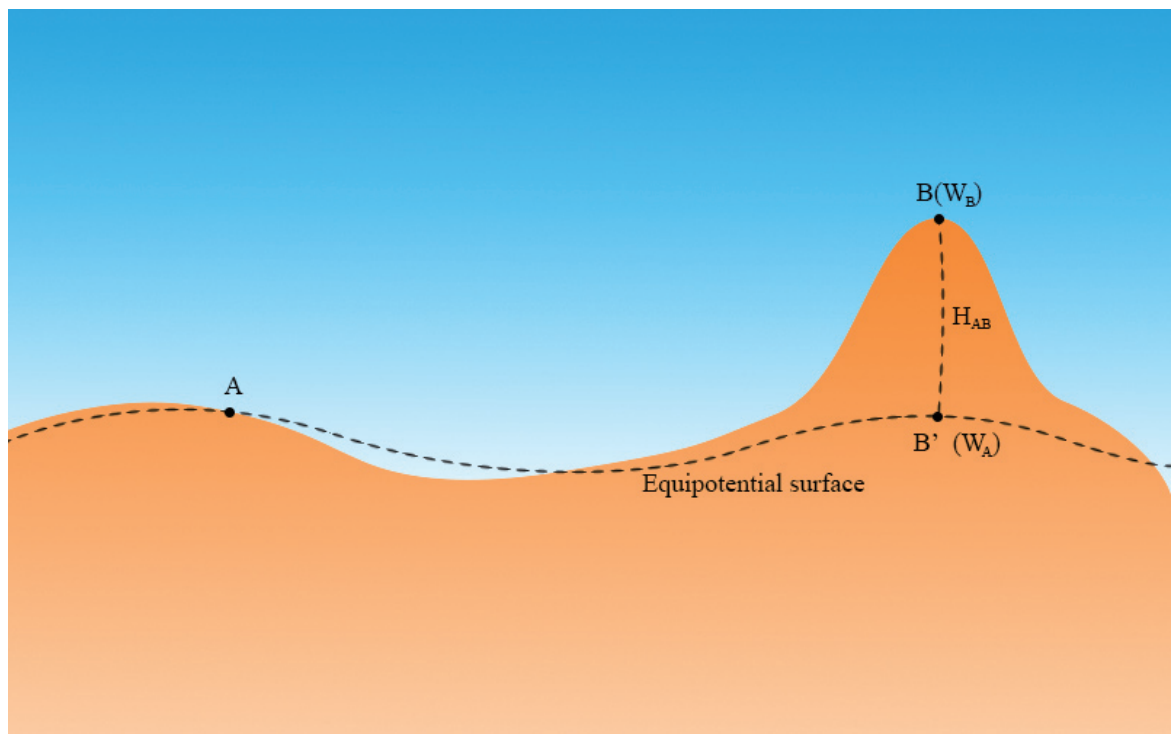

Fig. 1. Principle of determining the height difference between two points. Point $B$ ' is on the plumb line of point $B$, and points $A$ and $B$ ' are on a same equipotential surface (horizontal dashed curve). Therefore, the length of plumb line segment (vertical dashed curve) denotes the height difference between $A$ and $B$. 


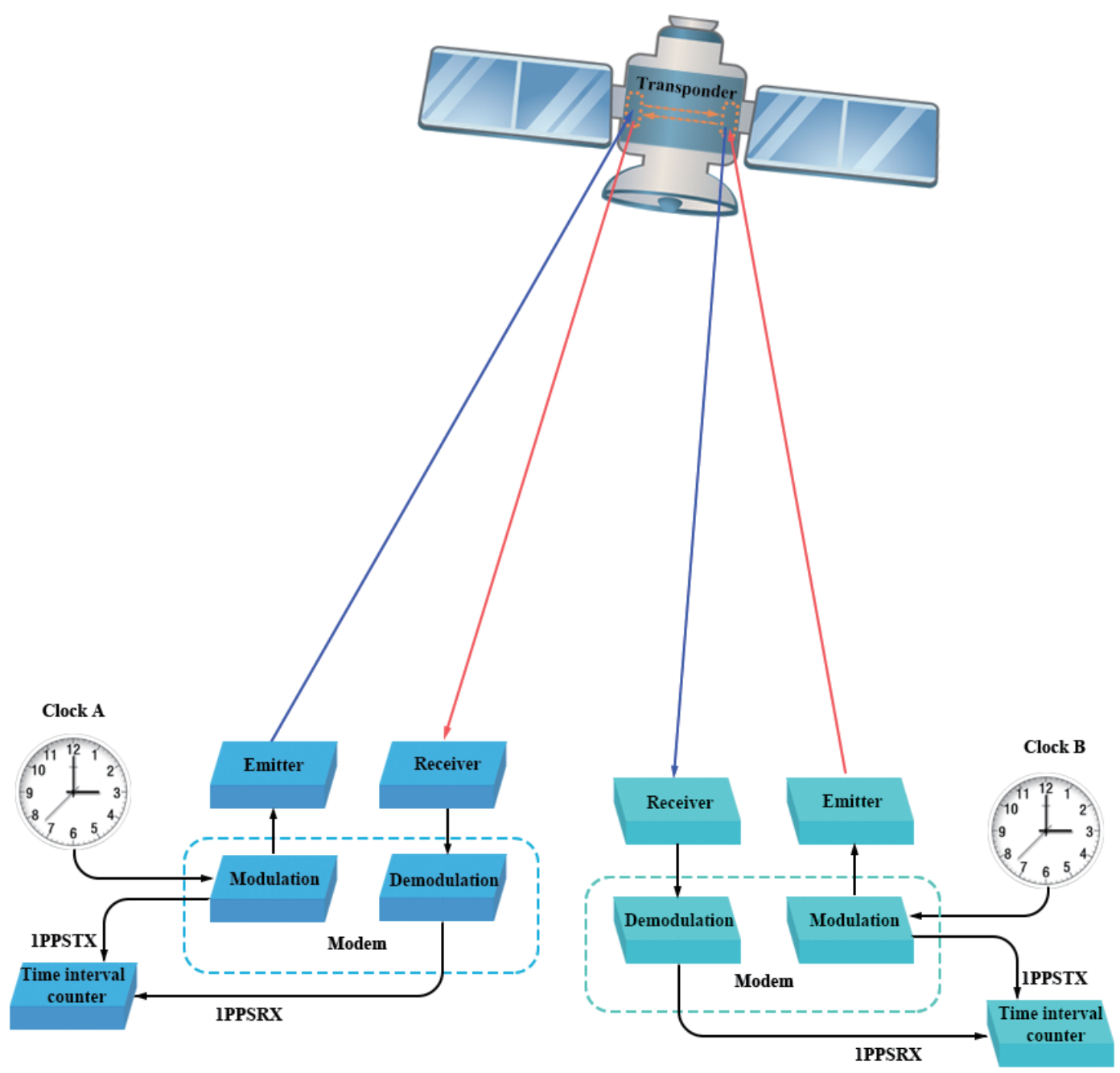

Fig. 2. Scheme of the TWSTFT-GD (two-way satellite time and frequency transfer used for geopotential determination) principle. The phase modulation is synchronized with the local clock, and the modem generates a one-pulse-per-second (1PPS) output, synchronous with the BPSK (Binary Phase Shift Keying) sequence, named 1PPSTX. This signal is the realization of a timescale named TS( $k)$. Each station uses a dedicated PRN code for its BPSK sequence in the transmitted signal. The receiving equipment generates the BPSK sequence of the remote stations and reconstitutes a 1PPS tick from the received signal, named 1PPSRX. The difference TI $(k)$ between the two 1PPS signals is measured by a time-interval counter (TIC) (modified after ITU-R 2010).

delay; $S P U(k)$ and $S P D(k)$, the signal path uplink and downlink delays, respectively; $S P T(k)$, the satellite path delay through the transponder; $S C U(k)$ and $S C D(k)$, the Sagnac corrections in the uplink and downlink, respectively.

\section{ERROR CORRECTION}

The observations include various errors, which should be cancelled or greatly reduced.

\subsection{Sagnac Effect Correction}

The Sagnac effect is caused by the Earth's rotation. Assuming a non-rotating Earth and a geostationary satellite, the signal is transmitted from the station to the satellite; thus, the route of the signal is not time-varying (Tseng et al.
2011). However, because the Earth rotates around its axis, the receiver located on Earth experiences uniform motion. Hence, during the signal propagation, the location of the receiver on the ground changes with respect to the satellite, and the corresponding influence is referred to as the Sagnac effect (Bidikar et al. 2016).

The Sagnac correction for a one-way path from satellite $s$ to ground station $k$ is given in a model that provides sufficient accuracy by the following expression (ITU-R 2010; Tseng et al. 2011; Bidikar et al. 2016):

$$
S C D(k)=\left(\Omega / c^{2}\right)[Y(k) X(s)-X(k) Y(s)]
$$

It is demonstrated that the Sagnac correction for the oneway path from ground station $k$ to satellite $s$ is exactly the 
opposite. Hence, we have the relation (ITU-R 2010):

$$
S C D(k)=-S C U(k)
$$

In our experiment, the two transmission antennas are quite close (within $5 \mathrm{~m}$ ); hence the effect of the position difference on Eq. (5) can be neglected. In this case, we have $S C D(1)=S C D(2)$ and $S C U(1)=S C U(2)$, and the total Sagnac correction as expressed by Eq. (4) reads

$$
-0.5[S C D(1)-S C U(1)]+0.5[S C D(2)-S C U(2)]=0
$$

\subsection{Ionospheric and Tropospheric Correction}

For the ionospheric correction, the uplink and downlink signals at each station differ in carrier frequency, and the total ionospheric delay is expressed as (ITU-R 2010; Rose et al. 2014)

$$
\left.\operatorname{SPU}(k)\right|_{i o n}-\left.\operatorname{SPD}(k)\right|_{i o n}=-40.3 \operatorname{TEC}(1 / c)\left(1 / f_{d}^{2}-1 / f_{u}^{2}\right)
$$

where TEC is the total electron content, namely the total number of free electrons along the signal propagation path, and the carrier frequencies $f_{u}$ and $f_{d}$ of the uplink and downlink signals are, respectively, 14.165 and $12.415 \mathrm{GHz}$. Similar to the situation described in section 3.1 , since the two transmission antennas are quite close (within $5 \mathrm{~m}$ ), the corresponding TEC values at each station are nearly the same. Hence, the ionospheric correction of each station is the same; namely, the effect of the ionosphere can be neglected in our experiment.

As for the troposphere, its influence on the difference between the up and down propagation delays is $<10 \mathrm{ps}$ (ITUR 2010), far less than the experimental precision. Hence, the troposphere's influence can also be neglected.

\subsection{Other Corrections}

The satellite delay difference, $S P T(1)$ - $S P T(2)$, is relevant to the satellite receiving antenna, transponder channel and transmission antenna, and the difference of the transmission and receiving section, $T X(k)-R X(k)$, is relevant to the up and down converters, modulator and demodulator (modem), amplifiers, antenna, cabling, etc. (ITU-R 2010).

In our experiment, the satellite delay difference is a constant value, $S P T(1)-S P T(2)=C_{1}$, and the time-varying effect of the ground station due to the Earth's rotation can be neglected. Conversely, the propagation delay of the two signals remains invariant because the two receivers are located at the same site (environmental factors such as temperature are the same). Furthermore, the satellite transponder delay of a geostationary satellite that varies with diurnal period- icity has no notable change (Liu et al. 2014), and hence, the satellite transponder delay of the two-way signals can be eliminated by a difference operation. Therefore, the difference of the transmission and receiving procedure in our experiment is also a constant value, which is expressed as

$0.5[T X(1)-R X(1)]-0.5[T X(2)-R X(2)]=C_{2}$

However, the relative position of the satellite used in our work is time-varying. ChinaSat 10 is the geostationary satellite used in our experiment, and its subastral point moves in orbit with a diurnal period (Wei 2013). Therefore, different emission times will induce different path delays, $S P U(1)$ and $S P U(2)$, to the satellite (ITU-R 2010). We consider that a path delay difference might exist in our measurement. Hence, Eq. (4) can be written as

$$
\begin{aligned}
T S(1)-T S(2)= & 0.5[T I(1)]-0.5[T I(2)] \\
& +C+a \sin \left(2 \pi t / T_{0}+\varphi\right)
\end{aligned}
$$

where $C$ denotes a constant delay, which consists of the satellite delay difference, cabling delay and so on, $T_{0}$ denotes the length of day, $a$ and $\varphi$ are constant values, denoting the amplitude and initial phase of the diurnal pattern, respectively.

\section{EXPERIMENTS}

To validate the geopotential determination based on GRT, we need to measure the elapsed time difference $\Delta t$ of two clocks at different stations.

There are two key problems in realizing this measurement. First, the clocks need to be of high stability to maintain a stable frequency standard. In our experiment, the instability of the frequency standard significantly limited the precision of the results. Second, we need a reliable time transfer technique to guarantee that when clock $\mathrm{C}_{\mathrm{A}}$ records a time duration $\Delta T$, clock $\mathrm{C}_{\mathrm{B}}$ will record a corresponding time duration $\Delta T^{\prime}$ that obeys the GRT with high accuracy (Shen and Shen 2015).

To solve the first problem, two sets of equipment were used in our experiment, each of which contains a hydrogen atomic clock whose relative nominal frequency stability is 5 $\times 10^{-15}$ in one day. As for the second problem, the TWSTFT technique was used. The TWSTFT technique is one of the most precise time transfer methods, other than the GNSS related techniques (Lin et al. 2014). In fact, direct time transfer such as fiber link has higher stability than time transfer via satellite, but satellite time transfer is better expected for long-distance height difference determination.

Our experiment was conducted in a building at the BIRMM, Beijing. As mentioned above, two hydrogen atomic clocks were used in our experiment; one is a 
non-transportable clock, $\mathrm{C}_{\mathrm{A}}$ (model H-MASTER VCH$1003 \mathrm{~A}$ ), and the other one is a transportable clock, $\mathrm{C}_{\mathrm{B}}$ (model BM2101-02).

The experiment was carried out in two continuous periods. Before these two periods, for the purpose of synchronization, we carried out a zero-baseline measurement from 6 December 2016 to 9 December 2016. As Fig. 3a shows, clocks $C_{A}$ and $C_{B}$ were both placed in a shielding room on floor B1 (ground floor) at position $P$. The shielding room was temperature-stabilized and controlled. Through connection cables, the two clocks, a pulse signal isolation amplifier (PSIA), a modulation and demodulation (modem) and a satellite antenna were connected.

In period 1, we carried out a geopotential difference measurement from 9 December 2016 to 27 December 2016 (as shown in Fig. 3b). During this process, $C_{B}$ was moved to fifth floor at position $Q$ (see Fig. 3), while $\mathrm{C}_{\mathrm{A}}$ remained fixed. The height difference between the ground floor and the fifth floor is $22.2 \mathrm{~m}$. Clocks $\mathrm{C}_{\mathrm{A}}$ and $\mathrm{C}_{\mathrm{B}}$ were connected by $150-\mathrm{m}$ length cables. Since there is a discontinuity of the time series in this period, we used a time series with 14-day length in practice.

In period 2, we carried out a zero-baseline measurement (shown in Fig. 3a) for comparison with the formal geo-potential difference measurement. Clocks $C_{A}$ and $C_{B}$ were both placed in a shielding room on floor $\mathrm{B} 1$ at position $P$. This zero-baseline measurement was performed from 27 December 2016 to 4 January 2017.

\section{DATA PROCESSING}

In our experiments, we obtained time-varying $T I(P)$ and $-T I(Q)$ every 1 second. At the beginning, due to a non-synchronization problem, the two time series did not align well with each other. Clock $\mathrm{C}_{\mathrm{A}}$ ran several seconds ahead of the clock $C_{B}$. However, this difference is insignificant. The key problem is that the running rates of the two clocks should be calibrated when they are located at the same level.

To make the data easy to process, we adopted the following three procedures:

(1) Linear interpolation is adopted to ensure that the data are time coherent;

(2) Data for a common time period are extracted to enable all data to be used in calculation;

(3) As the two signals at $P$ and $Q$ are not transmitted at exactly the same time, the series $T I(P)$ is moved to align with $-T I(Q)$.

After these procedures, we applied an averaging operation and obtained a series of data with a diurnal pattern (blue lines in Figs. 4a and b). To clearly show the coordinate values in these figures, we used the operation $\delta t=\Delta t-t_{0}$, where $t_{0}$ is the mean value of these observation data.

To remove periodic signals, we adopted a band-stop filter, specifically, a discrete-time FIR filter using a least- squares error minimization method. This band-stop filter eliminated the periodic signals which are not interested in this study. As shown in Figs. 4a and b, the blue lines show the observations without signal processing, and the purple curves denote the data obtained from the original observations after filtering. We see that at the beginning of the purple curves, there are large oscillations. This phenomenon is known as the "phase delay" and end effects, which occur for all kinds of FIR filters.

To obtain a first-order approximation, we used a linear function to fit the patterns after filtering (black lines in Figs. $4 \mathrm{a}$ and $\mathrm{b}$ ), and the parts of "phase delay" are ignored.

\section{RESULTS}

The slopes of the black lines as shown in Fig. 3 are reported in Table 1. To evaluate the frequency stability of each measurement, we adopted the modified Allan deviation (MADEV, Bregni 1997), which is estimated from a set of frequency measurements for averaging time $\tau$, expressed as (Allan et al. 1991; Bregni 1997)

$$
\sigma_{y}^{M}(\tau) \approx \sqrt{\frac{1}{2 \tau^{2} n^{2}(N-3 n+1)} \sum_{j=1}^{N-3 n+1}\left[\sum_{i=j}^{n+j-1}\left(x_{i+2 n}-2 x_{i+n}+x_{i}\right)\right]^{2}}
$$

where $\tau=n \tau_{0}, \tau_{0}$ is the sampling interval, $n$ is the number of the sampling interval from time $\tau_{0}$ to time $t$, and $N$ denotes the total number of the sampling interval of the whole time series.

The results are shown in Fig. 5 and Table 1.

By differencing the two slopes, we finally obtained

$$
\frac{\Delta t}{T}=k_{\text {geo }}-k_{\text {zero }}=-1.9060 \times 10^{-14}
$$

which indicates that clock $C_{B}$ runs faster than clock $C_{A}$ (fixed clock), with an uncertainty of

$\sigma=\sqrt{\sigma_{\text {geo }}^{2}+\sigma_{\text {zero }}^{2}}=6.47 \times 10^{-15}$

where $\sigma_{\text {geo }}$ and $\sigma_{\text {zero }}$ are the frequency stabilities of two measurements in period 1 and period 2, respectively.

Hence, the measured height difference is $\Delta H_{P Q}=$ $-\frac{\Delta t}{T} \frac{c^{2}}{g}=175.10 \mathrm{~m}$ with an accuracy of $59 \mathrm{~m}$.

\section{DISCUSSIONS AND CONCLUSIONS}

Our experimental results in period 1 (geo-potential difference measurement) provide a frequency stability 6.26 $\times 10^{-15}$ (shown in Fig. 5a), which is a little worse than the nominal frequency stability of the hydrogen atomic clocks 
(a)
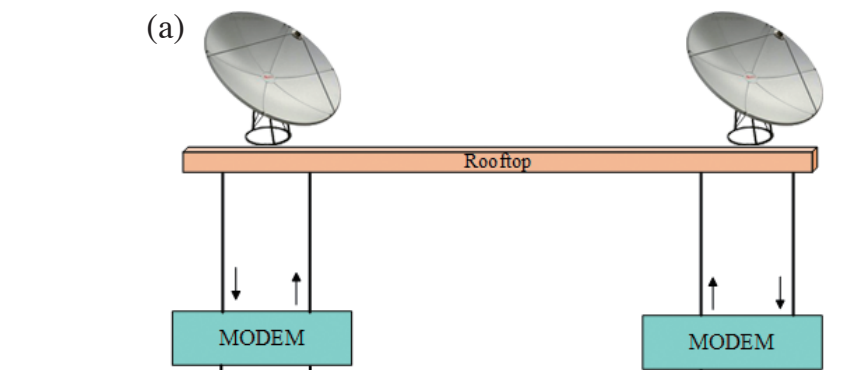

(b)

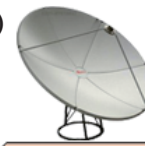

Floor $5(\mathrm{Q})$

(b)

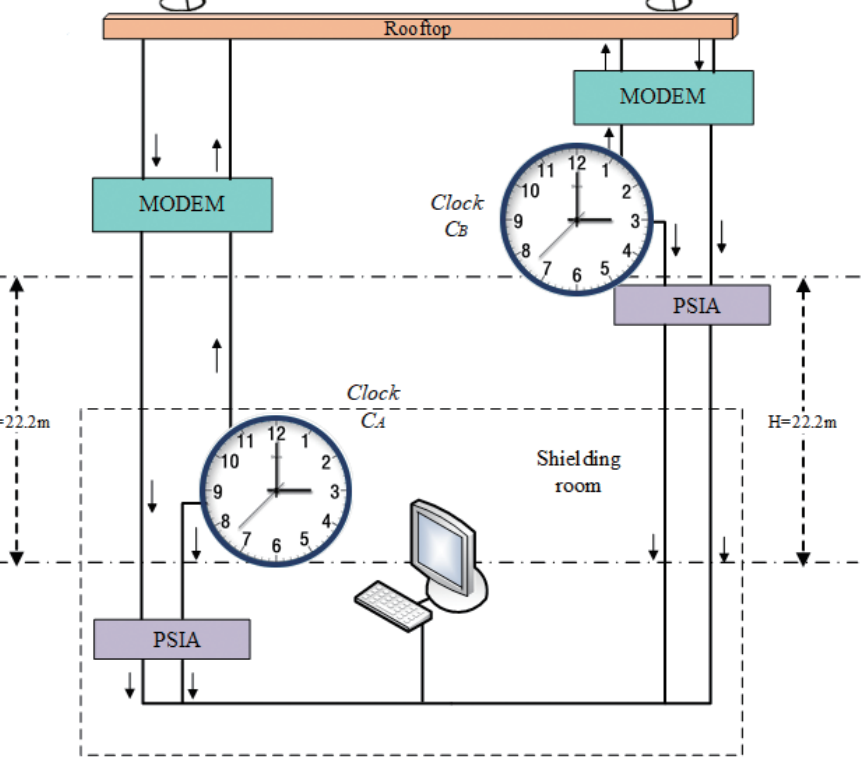

Fig. 3. (a) Zero-baseline measurement and (b) geopotential difference measurement. When the zero-baseline measurement was conducted, clocks $\mathrm{C}_{\mathrm{A}}$ and $\mathrm{C}_{\mathrm{B}}$ were both placed in a shielding room on floor $\mathrm{B} 1$ at position $P$. When the geopotential difference measurement was conducted, clock $\mathrm{C}_{\mathrm{B}}$ was placed on fifth floor at position $Q$, and clock $\mathrm{C}_{\mathrm{A}}$ was still placed on floor $\mathrm{B} 1$ at position $P$; they were connected with a satellite antenna through connection cables, modems, and a PSIA.

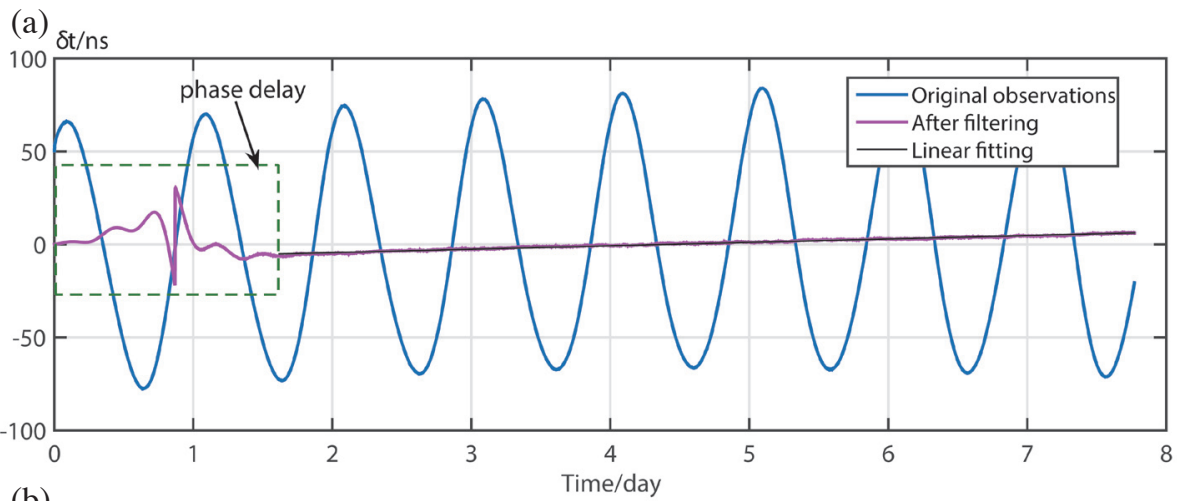

(b) $\delta$ t/ns

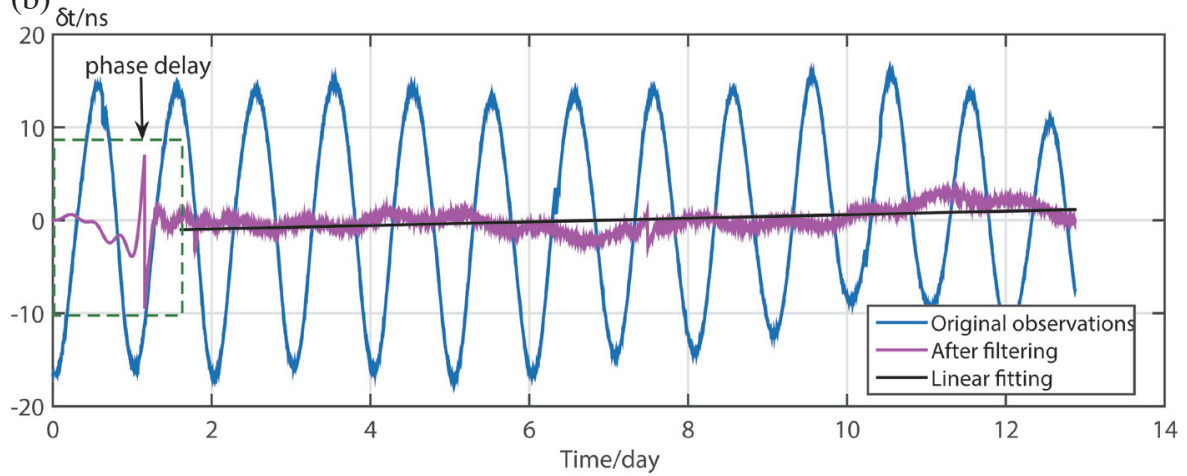

Fig. 4. (a) Data obtained by the zero-baseline measurement and (b) data obtained by the geopotential difference measurement. The blue curves show observation values without signal processing, and the purple curves are obtained after filtering. Based on the purple curves, we conducted linear fitting and obtained the black lines. 
Table 1. Slope and frequency stability of two measurements between the fixed clock $\mathrm{C}_{\mathrm{A}}$ and the transportable clock $C_{B}$.

\begin{tabular}{c|cc}
\hline & Slope $(\boldsymbol{\Delta t} / \boldsymbol{T})$ & Long term frequency stability \\
\hline Geo-potential difference measurement in 14 days (period 1) & $2.23 \times 10^{-15}$ & $6.26 \times 10^{-15}$ \\
Zero-baseline measurement in 8 days (period 2) & $2.129 \times 10^{-14}$ & $1.66 \times 10^{-15}$ \\
\hline
\end{tabular}
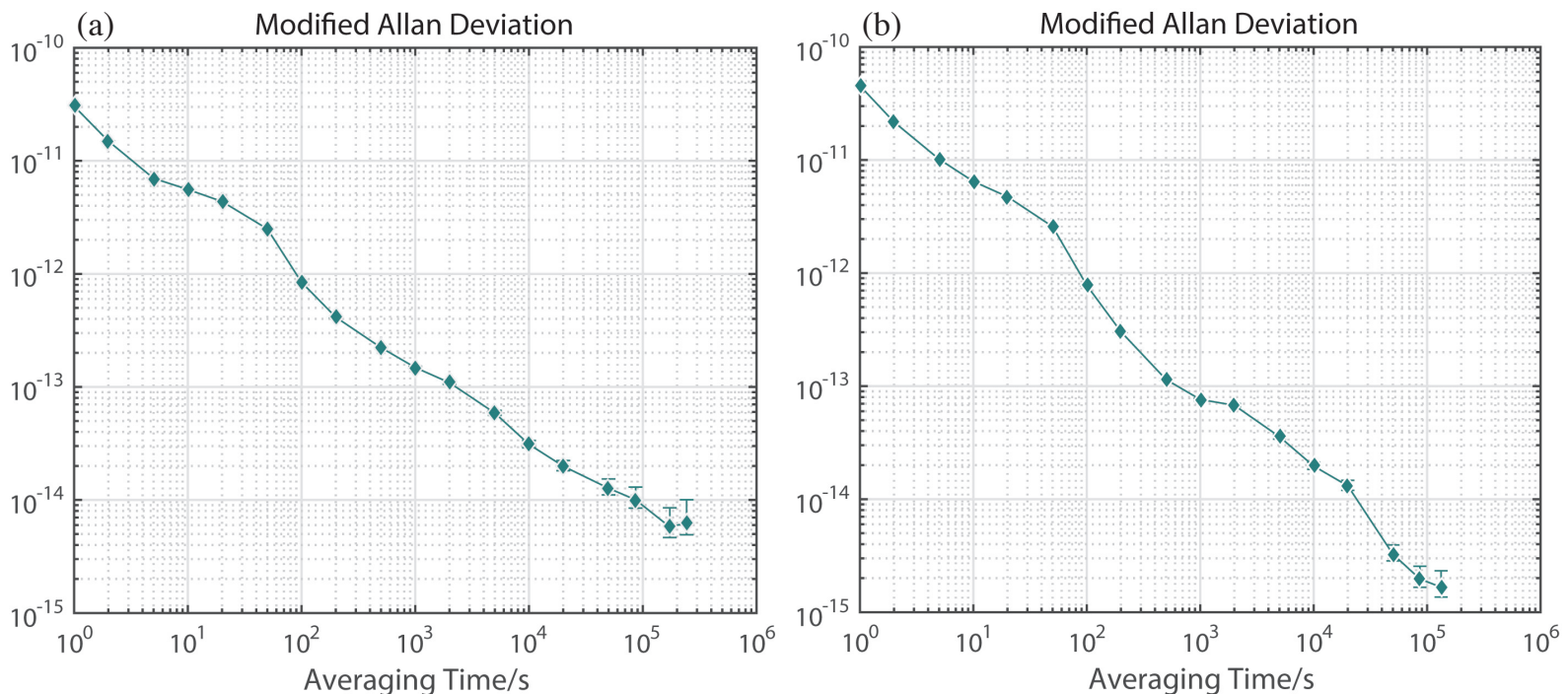

Fig. 5. (a) Frequency stability of data obtained by the geopotential difference measurement in period 1 and (b) frequency stability of data obtained by the zero-baseline measurement in period 2. These data are evaluated using the MADEV. The error bars indicate $1 \sigma$ uncertainties.

$\left(5 \times 10^{-15}\right.$ in one day). In period 2 (zero-baseline measurement), the experimental frequency stability is $1.66 \times 10^{-15}$ (shown in Fig. 5b), which is better than the nominal frequency stability of the hydrogen atomic clocks.

We obtained a height difference $175 \mathrm{~m}$ with an accuracy of $59 \mathrm{~m}$. Though the bias between the measured height difference and the true value $22 \mathrm{~m}$ is $153 \mathrm{~m}$, concerning the observation accuracy level of $59 \mathrm{~m}$, the results are acceptable. The results might be also influenced by other factors. One main concerning is that the environments are not well controlled. In fact, the temperature has a significant influence on the running rate of an atomic clock (Ascarrunz et al. 1996, 1997). When the clocks are placed on floor B1 at position $P$, there is a shielding room; thus, the temperature and other parameters are stable. However, on fifth floor at position $Q$, the environmental factors are not well controlled. In this case, the slope obtained (see Table 1) might not be reliable. The potential errors of the TWSTFT and the limitations by the observed diurnal variation may be also important factors besides the clock performance. In our experiment, the amplitude of the diurnal variation is much larger than the linear part (especially in zero-baseline measurement), significantly affecting the gravitational redshift term. Moreover, the variation of the satellite's trajectory also greatly influences the results. As mentioned in section 3.3, the subastral point of ChinaSat 10 moves in an orbit with a diurnal period. In addition, the orbit is not fixed, which varies every day.

Here we used two hydrogen atomic clocks and TWSTFT technique to determine the geopotential difference. Results show that the precision of the TWSTFT that we use in this study is in the same accuracy level as the GNSS commonview measurements provided by Kopeikin et al. (2016). Our experimental results are preliminary. Further experiments and investigations are needed to improve the results.

Acknowledgements Thanks are given to Beijing Institute of Radio Metrology and Measurement (BIRMM) for providing experiment platform, and especially to the Frequency Measurement Laboratory of BIRMM for providing satellite two-way time transfer system. We would like to express our sincere thanks to two anonymous reviewers for their valuable comments and suggestions, which improved the manuscript. This study is supported by the NSFCs (grant Nos. 41631072, 41721003, 41804012, 41574007), the Discipline Innovative Engineering Plan of Modern Geodesy and Geodynamics (grant No. B17033), DAAD Thematic Network Project (grant No. 57173947), and ISSI 2017 Supporting Project. 


\section{REFERENCES}

Allan, D. W., M. A. Weiss, and J. L. Jespersen, 1991: A frequency-domain view of time-domain characterization of clocks and time and frequency distribution systems. Proceedings of the 45th Annual Symposium on Frequency Control 1991, IEEE, 667-678, doi: 10.1109/freq.1991.145966. [Link]

Alley, C. O., 1979: Relativity and clocks. 33rd Annual Symposium on Frequency Control, IEEE, doi: 10.1109/ freq.1979.200296. [Link]

Ascarrunz, F. G., S. R. Jefferts, and T. E. Parker, 1996: Environmental effects on errors in two-way time transfer. Proceedings of 20th Biennial Conference on Precision Electromagnetic Measurements, IEEE, 518-519, doi: 10.1109/cpem.1996.547327. [Link]

Ascarrunz, F. G., S. R. Jefferts, and T. E. Parker, 1997: Earth station errors in two-way time and frequency transfer. IEEE Trans. Instrum. Meas., 46, 205-208, doi:10.1109/19.571813. [Link]

Bauch, A., 2015: Time and frequency comparisons using radiofrequency signals from satellites. Compt. Rendus Phys., 16, 471-479, doi: 10.1016/j.crhy.2015.02.006. [Link]

Bidikar, B., G. S. Rao, and L. Ganesh, 2016: Sagnac effect and SET error based pseudorange modeling for GPS applications. Procedia Computer Science, 87, 172177, doi: 10.1016/j.procs.2016.05.144. [Link]

Bjerhammar, A., 1985: On a relativistic geodesy. Bull. géodésique, 59, 207-220, doi: 10.1007/BF02520327. [Link]

Bloom, B. J., T. L. Nicholson, J. R. Williams, S. L. Campbell, M. Bishof, X. Zhang, W. Zhang, S. L. Bromley, and J. Ye, 2014: An optical lattice clock with accuracy and stability at the 10-18 level. Nature, 506, 71-75, doi: 10.1038/nature 12941. [Link]

Bregni, S., 1997: Clock stability characterization and measurement in telecommunications. IEEE Trans. Instrum. Meas., 46, 1284-1294, doi: 10.1109/19.668274. [Link]

Chou, C.W.,D.B.Hume, T.Rosenband, and D.J.Wineland, 2010: Optical clocks and relativity. Science, 329, 16301633, doi: 10.1126/science.1192720. [Link]

Delva, P. and J. Lodewyck, 2013: Atomic clocks: New prospects in metrology and geodesy. arXiv preprint arXiv:1308.6766.

Dittus, H., C. Lämmerzahl, A. Peters, and S. Schiller, 2007: OPTIS - A satellite test of special and general relativity. Adv. Space Res., 39, 230-235, doi: 10.1016/j. asr.2007.02.074. [Link]

Einstein, A., 1915: Die feldgleichungen der gravitation. Sitzungsberichte der Königlich Preußischen Akademie der Wissenschaften (Berlin), 844-847.

Flury, J., 2016: Relativistic geodesy. J. Phys. Conf. Ser.,
723, 012051, doi: 10.1088/1742-6596/723/1/012051. [Link]

Fujieda, M., D. Piester, T. Gotoh, J. Becker, M. Aida, and A. Bauch, 2014: Carrier-phase two-way satellite frequency transfer over a very long baseline. Metrologia, 51, 253-262, doi: 10.1088/0026-1394/51/3/253. [Link]

Fujieda, M., T. Gotoh, and J. Amagai, 2016: Advanced two-way satellite frequency transfer by carrier-phase and carrier-frequency measurements. J. Phys. Conf., 723, 012036, doi: 10.1088/1742-6596/723/1/012036. [Link]

Grotti, J., S. Koller, S. Vogt, S. Häfner, U. Sterr, C. Lisdat, H. Denker, C. Voigt, L. Timmen, A. Rolland, F. N. Baynes, H. S. Margolis, M. Zampaolo, P. Thoumany, M. Pizzocaro, B. Rauf, F. Bregolin, A. Tampellini, P. Barbieri, M. Zucco, G. A. Costanzo, C. Clivati, F. Levi, and D. Calonico, 2018: Geodesy and metrology with a transportable optical clock. Nat. Phys., 14, 437441, doi: 10.1038/s41567-017-0042-3. [Link]

Hafele, J. C. and R. E. Keating, 1972: Around-the-world atomic clocks: Observed relativistic time gains. Science, 177, 168-170, doi: 10.1126/science.177.4044.168. [Link]

Hinkley, N., J. A. Sherman, N. B. Phillips, M. Schioppo, N. D. Lemke, K. Beloy, M. Pizzocaro, C. W. Oates, and A. D. Ludlow, 2013: An atomic clock with 10-18 instability. Science, 341, 1215-1218, doi: 10.1126/science.1240420. [Link]

Hofmann-Wellenhof, B. and H. Moritz, 2006: Physical Geodesy, Springer, Vienna, doi: 10.1007/978-3-21133545-1. [Link]

ITU-R, 2010: The operational use of two-way satellite time and frequency transfer employing pseudorandom noise codes. TF Series: Time signals and frequency standards emissions, Recommendation ITU-R TF.11533 (03/2010), International Telcomunication Union. Available at http://www.itu.int/rec/r-rec-tf.1153/en.

JCGM 100, 2008: Evaluation of measurement data - Guide to the expression of uncertainty in measurement. International Bureau of Weights and Measures (BIPM), JCGM. Available at https://www.bipm.org/utils/common/documents/jcgm/JCGM 1002008 E.pdf.

Jiang, Z., H. Konate, and W. Lewandowski, 2013: Review and preview of two-way time transfer for UTC generation - From TWSTFT to TWOTFT. 2013 Joint European Frequency and Time Forum \& International Frequency Control Symposium (EFTF/IFC), Prague, Czech Republic, IEEE, doi: 10.1109/eftfifc.2013.6702103. [Link]

Kirchner, D., 1991: Two-way time transfer via communication satellites. Proceedings of the IEEE, 79, 983-990, doi: 10.1109/5.84975. [Link]

Kong, X., W. Shen, and S. Zhang, 2016: Determination of the geopotential and orthometric height difference 
based on the two way satellite time and frequency transfer observations. Geomatics and Information Science of Wuhan Univers, 41, 969-973, doi: 10.13203/j. whugis20140296. [Link]

Kopeikin, S. M., V. F. Kanushin, A. P. Karpik, A. S. Tolstikov, E. G. Gienko, D. N. Goldobin, N. S. Kosarev, I. G. Ganagina, E. M. Mazurova, A. A. Karaush, and E. A. Hanikova, 2016: Chronometric measurement of orthometric height differences by means of atomic clocks. Gravitation and Cosmology, 22, 234-244, doi: 10.1134/s0202289316030099. [Link]

Krisher, T.P., D. D. Morabito, and J. D. Anderson, 1993: The Galileo solar redshift experiment. Phys. Rev. Lett., 70, 2213-2216, doi: 10.1103/physrevlett.70.2213. [Link]

Lin, H. T., Y. J. Huang, W. H. Tseng, C. S. Liao, and F. D. Chu, 2014: The TWSTFT links circling the world. 2014 IEEE International Frequency Control Symposium (FCS), Taipei, Taiwan, IEEE, doi: 10.1109/ fcs.2014.6859962. [Link]

Lisdat, C., G. Grosche, N. Quintin, C. Shi, S. M. F. Raupach, C. Grebing, D. Nicolodi, F. Stefani, A. Al-Masoudi, S. Dörscher, S. Häfner, J.-L. Robyr, N. Chiodo, S. Bilicki, E. Bookjans, A. Koczwara, S. Koke, A. Kuhl, F. Wiotte, F. Meynadier, E. Camisard, M. Abgrall, M. Lours, T. Legero, H. Schnatz, U. Sterr, H. Denker, C. Chardonnet, Y. Le Coq, G. Santarelli, A. Amy-Klein, R. Le Targat, J. Lodewyck, O. Lopez, and P.-E. Pottie, 2016: A clock network for geodesy and fundamental science. Nat. Comm., 7, 12443, doi: 10.1038/ncomms12443. [Link]

Liu, J., Z. Li, X. Yang, J. Ou, S. Zhong, B. Sun, J. Wang, Y. Kong, and Z. He, 2014: Variation of satellite transponder delay. Chin. Sci. Bull., 59, 2568-2573, doi: 10.1007/s11434-014-0342-2. [Link]

Mai, E. and J. Müller, 2013: General remarks on the potential use of atomic clocks in relativistic geodesy. ZFV - Zeitschrift für Geodäsie, Geoinformation und Landmanagement, 138, 257-266.

Müller, J., D. Dirkx, S. M. Kopeikin, G. Lion, I. Panet, G. Petit, and P. N. A. M. Visser, 2018: High Performance Clocks and Gravity Field Determination. Space Sci. Rev., 214, doi: 10.1007/s11214-017-0431-z. [Link]

Pavlis, N. K. and M. A. Weiss, 2003: The relativistic redshift with $3 \times 10^{-17}$ uncertainty at NIST, Boulder, Colorado, USA. Metrologia, 40, 66-73.

Pound, R. V. and G. A. Rebka, 1959: Gravitational red-shift in nuclear resonance. Phys. Rev. Lett., 3, 439-441, doi: 10.1103/physrevlett.3.439. [Link]

Pound, R. V. and G. A. Rebka, 1960a: Attempts to detect resonance scattering in $\mathrm{Zn}^{67}$; The effect of zero-point vibrations. Phys. Rev. Lett., 4, 397-399, doi: 10.1103/ physrevlett.4.397. [Link]

Pound, R. V. and G. A. Rebka, 1960b: Variation with temperature of the energy of recoil-free gamma rays from solids. Phys. Rev. Lett., 4, 274-275, doi: 10.1103/physrevlett.4.274. [Link]

Pound, R. V. and J. L. Snider, 1965: Effect of gravity on gamma radiation. Phys. Rev., 140, B788-B803, doi: 10.1103/physrev.140.b788. [Link]

Riehle, F., 2017: Optical clock networks. Nat. Photon., 11, 25-31, doi: 10.1038/nphoton.2016.235. [Link]

Rose, J. A. R., R. J. Watson, D. J. Allain, and C. N. Mitchell, 2014: Ionospheric corrections for GPS time transfer. Radio Sci., 49, 196-206, doi: 10.1002/2013rs005212. [Link]

Shen, W. B., 2013a: Orthometric height determination based upon optical clocks and fiber frequency transfer technique. 2013 Saudi International Electronics, Communications and Photonics Conference, IEEE, doi: 10.1109/siecpc.2013.6550987. [Link]

Shen, W. B., 2013b: Orthometric height determination using optical clocks. EGU General Assembly 2013, held 7-12 April, 2013 in Vienna, Austria.

Shen, W. B., 2017: Unification of world height system at one centimeter level using ultra-high precise timefrequency signal links between a satellite and two ground datum stations. 19th EGU General Assembly, EGU2017, proceedings from the conference held 2328 April, 2017 in Vienna, Austria.

Shen, W. B. and Z. Peng, 2012: Gravity potential determination using remote optical fiber. International Symposium on Gravity, Geoid and Height Systems (GGHS), Venice, Italy.

Shen, W. B., D. Chao, and B. Jin, 1993: On relativistic geoid. Bollettino di geodesia e scienze affini, 52, 207-216.

Shen, W. B., J. Ning, D. Chao, and J. Liu, 2009: A proposal on the test of general relativity by clock transportation experiments. Adv. Space Res., 43, 164-166, doi: 10.1016/j.asr.2008.04.001. [Link]

Shen, W. B., J. Ning, J. Liu, J. Li, and D. Chao, 2011: Determination of the geopotential and orthometric height based on frequency shift equation. Nat. Sci., 3, 388396, doi: 10.4236/ns.2011.35052. [Link]

Shen, Z. and W. B. Shen, 2015: Geopotential difference determination using optic-atomic clocks via coaxial cable time transfer technique and a synthetic test. Geodesy Geodyn., 6, 344-350, doi: 10.1016/j.geog.2015.05.012. [Link]

Shen, Z., W. B. Shen, and S. Zhang, 2016a: Formulation of geopotential difference determination using opticalatomic clocks onboard satellites and on ground based on Doppler cancellation system. Geophys. J. Int., 206, 1162-1168, doi: 10.1093/gji/ggw198. [Link]

Shen, Z., W. B. Shen, and S. Zhang, 2016b: Simulation experiments of gravitational potential determination using clocks onboard satellite and on ground. EGU General Assembly 2016, held 17-22 April, 2016 in Vienna Austria. 
Shen, Z., W. B. Shen, and S. Zhang, 2017: Determination of gravitational potential at ground using optical-atomic clocks on board satellites and on ground stations and relevant simulation experiments. Surv. Geophys., 38, 757-780, doi: 10.1007/s10712-017-9414-6. [Link]

Shen, Z., W. B. Shen, and S. Zhang, 2018a: World height unification using satellite frequency signal transmission approach. IX Hotine-Marussi Symposium, June 18-22, Rome.

Shen, Z., W. B. Shen, Z. Peng, T. Liu, S. Zhang, and D. Chao, 2018b: Formulation of determining the gravity potential difference using ultra-high precise clocks via optical fiber frequency transfer technique. J. Earth Sci., doi: 10.1007/s12583-018-0834-0. [Link]

Tseng, W.-H., K.-M. Feng, S.-Y. Lin, H.-T. Lin, Y.-J. Huang, and C.-S. Liao, 2011: Sagnac effect and diurnal correction on two-way satellite time transfer. IEEE Trans. Instrum. Meas., 60, 2298-2303. doi:10.1109/ tim.2010.2095542. [Link]

Turneaure, J. P., C. M. Will, B. F. Farrell, E. M. Mattison, and R. F. C. Vessot, 1983: Test of the principle of equivalence by a null gravitational red-shift experiment. Phys. Rev. D, 27, 1705-1714, doi: 10.1103/physrevd.27.1705. [Link]

Ushijima, I., M. Takamoto, M. Das, T. Ohkubo, and H. Katori, 2015: Cryogenic optical lattice clocks. Nat. Photon., 9, 185-189, doi: 10.1038/nphoton.2015.5. [Link]

Vessot, R. F. C., M. W. Levine, E. M. Mattison, E. L. Blomberg, T. E. Hoffman, G. U. Nystrom, B. F. Farrel, R. Decher, P. B. Eby, C. R. Baugher, J. W. Watts, D. L. Teuber, and F. D. Wills, 1980: Test of relativistic gravitation with a space-borne hydrogen maser. Phys. Rev. Lett., 45, 2081-2084, doi: 10.1103/physrevlett.45.2081. [Link]

Wei, P., 2013: Method of common-view time transfer with transfer mode based on geostationary communication satellite. Ph.D. Thesis, National Time Service Center.

Zhang, V. S. and T. E. Parker, 2009: Two-way satellite time and frequency transfer using $1 \mathrm{MChip} / \mathrm{s}$ codes. 41st Annual Precise Time and Time Interval (PTTI) Systems and Applications Meeting, Santa Ana Pueblo, NM. 